\title{
Situated and embodied interactions for symbiotic and inclusive societies
}

\author{
Osamu Katai $\cdot$ Toyoaki Nishida $\cdot$ Renate Fruchter
}

Published online: 21 October 2010

(C) Springer-Verlag London Limited 2010

\section{Introduction}

Our daily life is occupied by a large variety of social actions toward other agents such as asking questions, making promises, expressing gratitude, making offers, making requests, giving warnings, making apologies, and so on, as suggested by speech act theory. We do so to live a better life by collaborating or coordinating our behavior with others. Social interactions are not monolithic. Usually, the above actions consist of smaller pieces of embodied behavior such as hand gestures or eye gaze in addition to spoken utterances. At the same time, they form a longitudinal process of human network development with a varying strength of trust.

Based on a bilateral definition of social intelligence, that is, an individual's ability to manage relationships with other agents and act wisely in a social situation, and an attribute of a group of people to manage complexity and learn from experiences as a function of a well-designed social structure (Nishida 2001), researchers in social intelligence design have been attempting to uncover the detailed principles and mechanisms as well as incorporate insights into artificial systems at different levels of abstraction.

O. Katai · T. Nishida $(\square)$

Graduate School of Informatics,

Kyoto University, Kyoto, Japan

e-mail: nishida@i.kyoto-u.ac.jp

O. Katai

e-mail: okatai@gaia.eonet.ne.jp

R. Fruchter

PBL Lab, Department of Civil and Environmental Engineering,

Stanford University, Stanford, CA 94305-4020, USA

e-mail: fruchter@stanford.edu
The first social intelligence design workshop held in 2001 identified five subjects in social intelligence design: theoretical aspects, methods of establishing the social context, embodied conversational agents, collaboration design, and public discourse. In the following workshops, further topics have been explored, such as mediated communication and interaction (Fruchter et al. 2005), natural interaction (Nijholt and Nishida 2006), collaboration technology and multidisciplinary perspectives (Fruchter et al. 2007), evaluation and modeling (Miura and Matsumura 2009), ambient intelligence (Nijholt et al. 2009), and designing socially aware interaction (Cavallin et al. 2010).

This special issue contains a selection of papers presented at the 8th International Workshop on Social Intelligence Design held in Kyoto in November 2009. This workshop focused on situated and embodied interactions for symbiotic and inclusive societies acknowledging the need for designing tools, procedures, techniques, and frameworks to improve interactions by addressing socially or ecologically related factors.

\section{Social intelligence design in a structured view}

Social intelligence design is studied at three levels of social granularity ranging from the macroscopic to the microscopic (Nishida 2010). At each level, social intelligence is discussed from the design, analytic, and synthetic views. The design view is holistic, attempting at realizing better design by harmonically incorporating organization, system, and environment based on a good understanding of social intelligence and interaction at a given level, while the analytic and synthetic views aim at uncovering and implementing principles underlying social interactions at that level using novel techniques. 
Social intelligence design on the macroscopic level aims at understanding and supporting communities where knowledge evolves as a result of interaction among members. Community knowledge management should recognize best practice in a community and enhance the knowledge spiral between formal and tacit knowledge. To foster participation, which is critical at this level, further issues need to be considered such as controlled anonymity in communication, social awareness, conviviality for promoting values such as empathy, reciprocity, social cohesion, inclusiveness, and participation, and social improvisational acts toward communication aiming at creative and humanistic communities. Tools have been presented for supporting large-scale discussions in a community. Those tools allow the user to extract social concern or grasp who else is present and which topics are being discussed. They are expected to strengthen the legitimacy and rationality of the democratic decision-making processes by using CMC tools to inspire and guide large-scale political debates.

Analytic approaches were taken to gain better understanding by investigating task-related domain-specific knowledge in information retrieval or by analyzing discussion $\operatorname{logs}$ in a large forum. Interesting insights have been obtained on culture dependency, self-efficacy (the cognition about one's capabilities to produce designated levels of performance), and placed-based presence (presence enhanced with concepts from the spatial model of interaction).

Social intelligence design at the mesoscopic level focuses on collaboration through structured interactions in a group. Major issues include design and analysis of global teamwork, collaboration support tools, and meeting support and smart meeting rooms. In the network era, workspaces are enhanced with information and communication technologies. According to a study on how people work together at a distance using a collaborative argumentative graph, the number of turning actions (those changing the structure of an argumentative graph) greatly varies between the face-to-face and remote conditions, while that of building actions (those contributing new information on the screen) does not. To allow people to flexibly interact with one another in a hybrid workplace, communication in the real life workplace needs to be analyzed in terms of physical space, communication space, and organizational space. Bricks (physical spaces)bits (electronic content)-interaction (the way people communicate with each other) was introduced to characterize collaboration support systems for global teamwork (Fruchter 2001). Collaboration support tools have been proposed to facilitate collaboration from different angles. They are based on a variety of ideas such as story telling as a vehicle for tacit-to-tacit knowledge transfer, multi- modal collaboration technology that supports global team work, an interactive platform to share ideas, knowledge, and insights in the form of concrete building projects, colocated interface for narration reconciliation in a conflict by making tangible the contributions and disagreements of participants and constraints imposed by the system to jointly perform some key actions on the story, and situated knowledge management. The virtual meeting room is proposed to improve remote meeting participation, to visualize multimedia data, and as an instrument for research into social interaction in meetings. Multi-agent systems fully automate a computational theory of social agents. Issues have been discussed on an interaction-oriented agent architecture and language that makes use of an interaction pattern, such as escape/intervention, social group dynamics in the behavior modeling of multi-agent systems, social judgment in multi-agent systems, and a multi-agent-based framework for simulating human and social behavior during emergency evacuation.

Analysis is as important as synthesis. Valuable insights have been obtained concerning the effects of long-term use of groupware: effects of scenarios on subjective usability evaluation across applications, the social relation between the moderator and interviewees, effects of the use of interaction media on social interactions, and the effect of the meeting assistant.

Social intelligence design at the microscopic level sheds light on fast interaction loops in social discourse. Major issues include interactive social assistants, analysis of nonverbal social behavior, and social artefacts. Systems have been presented that support conception and decision making of the user while online shopping, or support a jobhunter's decision making based on observations from their actual job-hunting process. Analysis of nonverbal social behavior will provide the insights needed to implement collaboration support systems or social artefacts. Methods have been presented for extracting structured information consisting of text and sketches from unstructured speech transcript archives that capture knowledge generated during informal communicative events through dialogue, sketching, and gestures, for exploiting gestures as a knowledge indexing and retrieval tool for unstructured digital video data, or for measuring gaze direction and facial features to detect hidden intention.

From an engineering point of view, social artefacts have been proposed that embody social intelligence to interact with people or other social agents. Typical subjects include mutual adaptation for establishing social interaction protocols, interactive perception to attenuate noise and unintended behavior components of the sensor signals, principles and methodologies for building social robots, design and evaluation of robot's gaze behavior, and investigating negative attitudes toward robots. 


\section{Situated and embodied interactions for symbiotic and inclusive societies}

The nine papers included in this special issue were selected and extended from the workshop presentations.

The first paper by Doi and Yamada (2010) comes from an invited talk on the future of embodied interactions for symbiotic and inclusive societies. It presents a roadmap for social innovation in the forthcoming 30 years by integrating social science and ICT, with a particular emphasis on technologies for converging the real and cyber worlds and those for integrating heterogeneous systems. A valuable feature of this paper is that it offers a proposal from an industrial sector about a potential contribution to the future global society.

The second paper by Askool and Nakata (2010) addresses identification of the factors that may influence adoption of SCRM (social customer relationship management). This paper proposes familiarity, caring behavior, sharing information, and perceived trustworthiness as key factors toward adoption of SCRM and shows a conceptual model of dependencies of relevant factors. In order to cope with the limited availability on SCRM, a scoping study consisting of Email-based interview followed by template analysis was conducted.

The third paper by Fruchter and Bosch-Sijtsema (2010) investigates social functions played by the WALL, a wall used as a large, public, physical display surface embedded in the center of the physical team workspace. The WALL, as the paper reports, acts as a mediator for individual reflection-in-action and team reflection-in-interaction, as well as serving as "social glue" both between individuals and between geographically distributed subgroups. As a result of data analysis and key observations, five interaction enablers and four interaction hindrances are identified.

The fourth paper by Fruchter and Courtier (2010) defines re-representation as a sequence of representations of the same concept using different communication channels and media. Fruchter and Courtier used the AEC Global Teamwork course offered in 2008-2009 as the test bed and investigated how the re-representation technique was to build common ground during multimodal and multimedia communicative events in cross-disciplinary, geographically distributed settings. The paper discusses that the observations may suggest several interesting principles such that capturing, sharing, and replaying of multimedia communicative events can facilitate common ground building and accelerate execution of action requests.

The fifth paper by Nishiyama et al. (2010) presents a decision model using the influence diagram that describes interdependencies among situation estimations, comfort estimations, and decision-making actions. The proposed model was implemented as an intelligent agent embedded in a home ambient intelligent system. The unique feature of the proposed approach is that the agent not only adapts to the user but also tries to make decisions to achieve an optimal balance between comfort and cost.

The sixth paper by Kaptein et al. (2010) reports the positive effects of social praise (positive feedback about the ongoing conversation) and mimicry (displaying matching behavior) on the perceived friendliness and intelligence of an artificial agent, based on a controlled laboratory experiment. They argue that both mimicry and social praise might be effective to realize a persuasive scenario and discuss how mimicry and praise might be implemented.

The seventh paper by Kiselev et al. (2010) discusses the integration of emotions with rationality. A target scenario of interactions between the user and a virtual technical support engineer (VTSE) was used to identify key issues in the use of affective computing in search for better client service in industrial applications. A prototype of VTSE was implemented, and a preliminary evaluation was made with a simplified model without an explicit emotion component. Based on the insights obtained with the preliminary research, the second model was proposed, which contains an explicit realization of a three-layered model of emotions, highly interconnected with other components of the system.

The eighth paper by Becker-Asano et al. (2010) studies socio-emotional aspects of laughter of humanoid robots. Based on online surveys and presentations at a Japanese and a German high school, they discuss how a humanoid robot might laugh in a situational context appropriate for laughter, whether people prefer certain styles of laughter for visually different kinds of humanoid robots, and whether the cultural background of the observer influences the perceived naturalness of a robot's laughter.

The ninth paper by Rutkowski et al. (2010) discusses the possibility of analyzing multimodal brain and peripheral bodily responses to affective stimuli. Some interesting findings were reported such as changes in multimodal state (EEG, EOG, and ECG) and within blood oxygenation signals correlations patterns and that the EEG and fNIRS channels present much stronger correlation pattern transition when the emotional stimuli are presented. The finding might be applied to BCI/BMI systems as well as socially aware intelligent environments or communication support systems.

\section{References}

Askool S, Nakata K (2010) A conceptual model for acceptance of social CRM systems based on a scoping study (in this special issue) 
Becker-Asano C et al (2010) Studying laughter in combination with two humanoid robots (in this special issue)

Cavallin H, Fruchter R, Nishida T (2010) The multiple faces of social intelligence design. AI Soc 25(2):141-143

Doi S, Yamada K (2010) Symbiotic technology for creating social innovation 30 years in the future (in this special issue)

Fruchter R (2001) Bricks and bits and interaction. In: Terano T et al (eds) Exploring new frontiers on artificial intelligence-selected papers from the first international workshops of Japanese society of artificial intelligence, Lecture Notes on Artificial Intelligence LNAI 2253 Springer Verlag, pp 35-42

Fruchter R, Bosch-Sijtsema P (2010) The WALL: participatory design workspace in support of creativity, collaboration, and socialization (in this special issue)

Fruchter R, Courtier R (2010) Building common ground in global teamwork through re-representation (in this special issue)

Fruchter R, Nishida T, Rosenberg D (2005) Understanding mediated communication: the social intelligence design (SID) approach. AI Soc 19(1):1-7

Fruchter R, Nishida T, Rosenberg D (2007) Mediated communication in action: a social intelligence design approach. AI Soc 22(2): 93-100

Kaptein M et al (2010) Two acts of social intelligence-the effects of mimicry and social praise on the evaluation of an artificial agent (in this special issue)
Kiselev A et al (2010) Towards incorporating emotions with rationality into a communicative virtual agent (in this special issue)

Miura A, Matsumura N (2009) Social intelligence design: a junction between engineering and social sciences. AI Soc 23(2):139-145

Nijholt A, Nishida T (2006) Social intelligence design for mediated communication. AI Soc 20(2):119-124

Nijholt A, Stock O, Nishida T (2009) Social intelligence design in ambient intelligence. AI Soc 24(1):1-4

Nishida T (2001) Social intelligence design — an overview. In: Terano T, Nishida T, Namatame A, Ohsawa Y, Tsumoto S, Washio T (eds) Exploring new frontiers on artificial intelligence-selected papers from the first international workshops of japanese society of artificial intelligence. LNAI 2253, Springer, Heidelberg, pp 3-10

Nishida T (2010) Social intelligence design for knowledge circulation. In: Kikuchi S, Sachdeva S, Bhalla S (eds) DNIS 2010, LNCS 5999, pp 122-142

Nishiyama T, Hibiya S, Sawaragi T (2010) Development of agent system based on decision model for creating an ambient space (in this special issue)

Rutkowski TM et al (2010) Emotional empathy transition patterns from human brain responses in interactive communication situations-brain computer and machine interactive interfacing approach (in this special issue) 